\title{
Reliability and Validity of the Korean Version of Children's Depression Inventory 2 Short Version as a Screening Tool: A Comparison With the Standard 28-Item Version
}

\author{
Yaehee Cho', Eun-Ho Lee ${ }^{2}$, Sang-Hwang Hong ${ }^{3}$, Yoo-Sook Joung ${ }^{1 凶}$, and Ji-Hae Kim ${ }^{1}$ \\ ${ }^{1}$ Department of Psychiatry, Samsung Medical Center, Sungkyunkwan University School of Medicine, Seoul, Republic of Korea \\ ${ }^{2}$ Depression Center, Department of Psychiatry, Samsung Medical Center, Sungkyunkwan University School of Medicine, Seoul, Republic of Korea \\ ${ }^{3}$ Department of Education, Chinju National University of Education, Jinju, Republic of Korea
}

Objective To investigate the reliability and validity of the Korean version of Children's Depression Inventory 2 Short Version (CDI 2:S) in comparison with its full-length version (CDI 2) as a screening tool for depressive youth.

Methods A total of 714 children from the community and 62 psychiatric patients were enrolled in this study. The Korean version of the Kiddie Schedule for Affective Disorders and Schizophrenia Present and Lifetime Version (K-SADS-PL-K) served as the reference standard for computing receiver operating characteristic (ROC) curves. To evaluate the ability of the CDI 2 and CDI 2:S to discriminate major depressive disorders, areas under the curves (AUCs) were compared. To investigate psychometric properties of the CDI 2:S, internal consistency was calculated and confirmatory factor analysis was conducted.

Results For the CDI 2, the cutoff at 20 yielded the best balance between sensitivity (83\%) and specificity (91\%). For the CDI 2:S, the cutoff point of 10 resulted in high sensitivity ( $82 \%$ ) and high specificity (93\%). The short form was proven to be as sensitive and specific as the CDI 2. Further analyses confirmed that the CDI 2:S also had good reliability and validity.

Conclusion The CDI 2:S, a sensitive and brief form of the CDI 2, may serve as a better option in time-constrained psychiatric settings.

Psychiatry Investig 2022;19(1):54-60

Keywords Child depression; CDI 2; CDI 2 short form; ROC analysis; Depression screening.

\section{INTRODUCTION}

Depression is a worldwide burden. ${ }^{1}$ Depression developed at younger ages has more debilitating effects on emotional, functional, and interpersonal domains than that emerged in adulthood..$^{2-5}$ Depressed youths tend to socially withdraw themselves, which consequently make them vulnerable to

\footnotetext{
Received: September 9, 2021 Revised: November 1, 2021

Accepted: November 15, 2021

$\triangle$ Correspondence: Ji-Hae Kim, PhD

Department of Psychiatry, Samsung Medical Center, Sungkyunkwan University School of Medicine, 81 Ilwon-ro, Gangnam-gu, Seoul 06351, Republic of Korea Tel: +82-2-3410-0931, Fax: +82-2-3410-0050

E-mail: Jihae0931.kim@samsung.com

$\triangle$ Correspondence: Yoo-Sook Joung, MD, PhD

Department of Psychiatry, Samsung Medical Center, Sungkyunkwan University School of Medicine, 81 Ilwon-ro, Gangnam-gu, Seoul 06351, Republic of Korea Tel: +82-2-3410-0930, Fax: +82-2-3410-0050

E-mail: yschoung@skku.edu

(a) This is an Open Access article distributed under the terms of the Creative Commons Attribution Non-Commercial License (https://creativecommons.org/licenses/bync/4.0) which permits unrestricted non-commercial use, distribution, and reproduction in any medium, provided the original work is properly cited.
}

severe depression..$^{6-9}$ The prevalence of child and adolescent depression has increased after the COVID-19 pandemic. According to a systematic review by Loades et al., ${ }^{10}$ children and adolescents are more likely to feel loneliness and have experienced dramatic disruptions to their daily lives after enforced social isolation. Considering that this early development stage is a critical period that shapes one's social competence and even long-term socioeconomic status, ${ }^{6}$ the prevention and management of child depression are necessary. However, research on depression assessment has mostly focused on the stage of adulthood, ${ }^{11,12}$ hindering the identification of depressed youth.

Self-report questionnaire is the most widely used method to detect a depressive disorder. ${ }^{13}$ Self-report inventories help clinicians to screen for depression and improve the recognition rates. The past studies have reported that several screening tools for child depression have high reliability, validity, and diagnostic utility. ${ }^{5}$ However, most of these tools (e.g., CES-D, BDI-II) were originally developed for use in adults, whereas 
the clinical profile is different between youth and adult. Although Reynolds Adolescent Depression Scale (RADS) was originally designed for detecting adolescent depression, it consists of 30 items and takes at least 10 minutes to complete, which decreases time efficiency in a time-constraint clinical setting. Thus, we propose a need for the evidence-based screening tool with two critical points: 1) originally developed for child and adolescent population, 2) short administration time.

The Children's Depression Inventory (CDI), which has been developed by Kovacs in 1992 is one of the most widely used scales for assessing depressive symptoms in children and adolescents. ${ }^{14-16}$ The CDI has been translated into more than 20 languages and currently in use worldwide. For instance, it has been validated in Arabic, ${ }^{17}$ Spanish, ${ }^{18}$ Dutch, ${ }^{19}$ German, ${ }^{20}$ Greek, ${ }^{21}$ Italian, ${ }^{22}$ Malaysian, ${ }^{23}$ Chinese, ${ }^{24}$ Japanese ${ }^{25}$ version. Also, the standardization studies have been conducted among diverse populations.

Since it was developed in 1992, Kovacs developed CDI 2 with updated normative sample for controlling birth cohort effect. In the CDI 2, some items were changed to better capture core symptoms of child depression and delivering clearer questions. ${ }^{26}$

To date, the CDI:S (Kovacs 1992) has been relatively widely used in clinical and research settings. ${ }^{20,27}$ However, the CDI 2:S has not been yet validated as it is relatively new. It was originally intended to screen youth at risk for depression. However, cut-off scores have not been proposed yet. Therefore, we conducted an initial yet unique study of the CDI 2:S and compared it with its full-length version (CDI 2) using a representative sample of Korean children and adolescents and clinical sample from a tertiary care hospital.

\section{METHODS}

\section{Participants}

We used 2 cohorts: community-based general population and clinical sample. The community sample were recruited from schools in 3 different cities (Seoul, Cheongju, and Jinju) in South Korea (for a detailed description) ${ }^{28}$ Participants were between 7 and 17 years old, possessing Korean reading and comprehension proficiency. They completed an informed consent and parental consent, and a sociodemographic questionnaire. Cases with missing demographic data or item responses were excluded in the analysis. In our study, we excluded the cases aged under 11 in community sample in order to match the age range of the clinical group. Since developmental stage is critical to emergence of depressive symptoms, we attempted to reduce selection bias.

Our clinical sample was recruited from the psychiatry at a tertiary care outpatient clinic from January 2021 to May 2021.
Outpatient children and adolescents (11-17 years) meeting the DSM-IV diagnostic criteria for major depressive disorder (MDD) and/or dysthymic disorder (DD) as determined by the Korean version of the Kiddie Schedule for Affective Disorders and Schizophrenia Present and Lifetime Version (K-SADS-PL-K) were enrolled in the study $(n=62)$. Informed consent was obtained from parents/children under protocols approved by the Institutional Review Board of Samsung Medical Center (IRB No. 2021-05-144-001). Patients were given the CDI 2 and asked to complete it on the day they visited the clinic. Total of 62 participants were included in the analyses. Thirty cases had single diagnosis of MDD (82\%), or DD (18\%). However, 20 participants had comorbid psychiatric disorders: attention-deficit/hyperactivity disorder $(60 \%)$, generalized anxiety disorder (20\%), and social phobia (20\%).

Whereas comorbid disorders with MDD or DD were allowed in order to increase statistical power with large sample size, MDD or DD was the primary disorder. Exclusion criteria for clinical samples were as follows: 1) full-scale intelligence quotient (FSIQ) lower than 50,2) patients with psychotic symptoms, 3) primarily diagnosed with bipolar I or II disorders. Eight participants were excluded from the study: 4 cases with psychotic symptoms, 3 cases diagnosed with bipolar ii disorders, 1 case with intellectual disability. Two cases were missing demographic information, thus were not included in the analyses.

\section{Measures}

\section{Children Depression Inventory 2 (CDI 2) and its short form (CDI 2:S)}

The CDI 2 consists of 28 items measuring depressive symptoms. Each item is scored on a scale of 0 (no symptom) to 2 (clinically significant), with higher score indicating more severe depressive symptoms (0-56). Participants choose the statement that best describes how they have felt for the past 2 weeks. The average time to complete the CDI 2 is within 15 minutes. The abbreviated version of the CDI 2 (CDI 2:S) is developed for a screening purpose. Kovacs omitted items with lower inter-items correlation, and item 'suicidal idea. The CDI 2:S consists of 12 items, and total scores range from 0 to 24 . The administration time for the CDI 2:S is less than 5 minutes.

\section{Korean version of the Kiddie Schedule for Affective Disorders and Schizophrenia Present and Lifetime Version (K-SADS-PL-K)}

K-SADS-PL-K has been used as a gold standard measure to confirm diagnosis of mood disorders according to DSMIV-R criteria. ${ }^{29}$ The K-SADS- PL-K has good reliability and validity for diagnosing psychiatric disorders in children and ad- 
olescents. ${ }^{30}$ We used the K-SADS-PL-K to confirm diagnoses. Eleven clinical psychologist residents interviewed the child and primary caregiver, and later confirmed by the three child/ adolescent psychiatrists interviewed the child and primary caregiver.

\section{Statistical analysis}

Descriptive statistics, reliability, concurrent validity analyses were performed using IBM SPSS 23 (IBM Corp., Armonk, NY, USA). To analyze all demographic data, we conducted descriptive statistics and frequency analysis. Diagnosis of major depressive disorder was performed according to the K-SADS-PL-K which served as an external criterion. Diagnostic utilities of the CDI 2 and the CDI 2:S was assessed by the area under the receiver operating characteristic (ROC) curve (AUC). If the value of the AUC was between 0.7 and 0.8 , it was considered acceptable. If $A U C \geq 0.8$, it was considered excellent. ${ }^{31}$ Then we used sensitivity, specificity, and predictive values to calculate the optimal cut-off score that could discriminate between the group with depression and the group without depression group. Optimal cut-off values were those that demonstrated a maximal Youden Index $(\mathrm{Y})^{32}$ indicating the highest combination of sensitivity and specificity. Additionally, assuming that the CDI 2:S could perform as well as the full-length version, we tested factorial validity of the CDI 2:S. The lavaan package in $\mathrm{R}$ statistical software ( $\mathrm{R}$ Studio, Boston, MA, USA) $)^{33}$ was used to conduct confirmatory factor analysis (CFA). The factorial structure was investigated using confirmatory factor analysis with weighted least squares estimator having a mean and variance (WLSMV). WLSMV is known to perform better than maximum likelihood method with fewer than five response categories as it does not assume normally distributed variables. ${ }^{34}$ Fit indices including comparative fit index (CFI), ${ }^{35}$ tucker lewis index (TLI), ${ }^{36}$ and root mean square error of approximation (RMSEA $)^{37}$ were used to evaluate the overall model fit. CFI and
TLI $\geq 0.90^{38}$ and RMSEA value of $\leq 0.08^{39}$ indicated a reasonably good fit.

\section{RESULTS}

\section{Demographic characteristics and diagnostic results}

Detailed information about the descriptive results of demographic characteristics and the scores of the CDI 2 and the CDI 2:S are presented in Table 1. Gender difference in depression is a common phenomenon. ${ }^{40}$ The results showed that regardless gender, depression group significantly scored high in the CDI 2 and the CDI 2:S (all p<0.001). We additionally conducted independent sample t-test analysis between genders in each group separately. Girls scored higher than boys for both the CDI 2 and the CDI 2:S in a community sample $\left(\mathrm{t}_{(709.19)}=\right.$ $-3.41, \mathrm{p}=0.001, \mathrm{t}_{(710.21)}=-3.20, \mathrm{p}=0.002$, respectively). However, in the depression group, a significant difference between gender is only observed in the CDI $2\left(\mathrm{t}_{(52)}=-2.11, \mathrm{p}=0.04\right)$. There was no significant difference in the CDI $2: \mathrm{S}$ across gender $\left(\mathrm{t}_{(52)}=\right.$ $-1.60, \mathrm{p}=0.12$ ).

\section{Diagnostic validity}

We conducted ROC analyses for the clinical group vs. community sample. Results of the ROC analysis are presented in Figure 1. For the CDI 2, the AUC value was 91.6 (95\% CI: 86.596.7) and the optimal screening cut-off score was 20 (Y=0.739) (Table 2). The positive predictive value (PPV) was $35.2 \%$ and the negative predictive value (NPV) was $98.6 \%$ in our sample. When we recalculated PPV and NPV according to disease prevalence in Korean child/adolescent sample (2.3\%; National Mental Health Survey, 2009), PPV and NPV were $8.8 \%, 96.1 \%$, respectively. For the CDI 2:S, the AUC value was 0.918 (95\% CI: 0.866-0.971) and the optimal screening cut-off score was 10 ( $\mathrm{Y}=0.749)$ (Table 3). In our clinical sample, PPV was $39.1 \%$ and NPV was $98.6 \%$. Considering a disease prevalence of $2.3 \%$, PPV and NPV were $9.6 \%$ and $95.8 \%$,

Table 1. Descriptive statistics of demographic characteristics and scores of Children Depression Inventory 2 (CDI 2) and its short-form (CDI 2:S)

\begin{tabular}{lccr}
\hline & Normal $(\mathrm{N}=714)$ & Depression group $(\mathrm{N}=54)$ & $\mathrm{p}$ value \\
\hline Age & $13.85(1.87)$ & $15.37(1.71)$ & 0.001 \\
Sex (male/female) $(\%)$ & $330(46.2) / 384(53.8)$ & $18(33.3) / 36(66.7)$ & 0.067 \\
CDI 2 & $10.43(6.75)$ & $28.74(10.08)$ & $<0.001$ \\
Male & $9.52(5.91)$ & $24.78(11.32)$ & $<0.001$ \\
Female & $11.21(7.32)$ & $30.72(8.91)$ & $<0.001$ \\
CDI 2:S & $4.78(3.29)$ & $13.43(4.54)$ & $<0.001$ \\
Male & $4.36(2.91)$ & $12.06(5.30)$ & $<0.001$ \\
Female & $5.14(3.56)$ & $14.11(4.01)$ & $<0.001$ \\
\hline
\end{tabular}

Standard deviation is shown in parentheses. $\mathrm{p}$ value for sex was calculated by chi-square test, $\mathrm{p}$ values for age, CDI 2 and CDI 2:S scores were calculated by independent sample t-test 
respectively.

\section{Internal consistency and factorial validity of the CDI 2:S}

Cronbach's alpha was 0.90 for the CDI 2 and 0.84 for the CDI 2:S. Correlation between the 28-item CDI 2 and the 12 item CDI 2:S was significant $(r=0.248, \mathrm{p}<0.001)$. The factorial validity of the CDI 2 was discussed in Kim et al..$^{28}$ Since no previous factorial analyses has been done on the short form, we tested the factorial validity of this short form. Results of CFA analysis indicated that the CDI 2:S was a unidimensional mea-

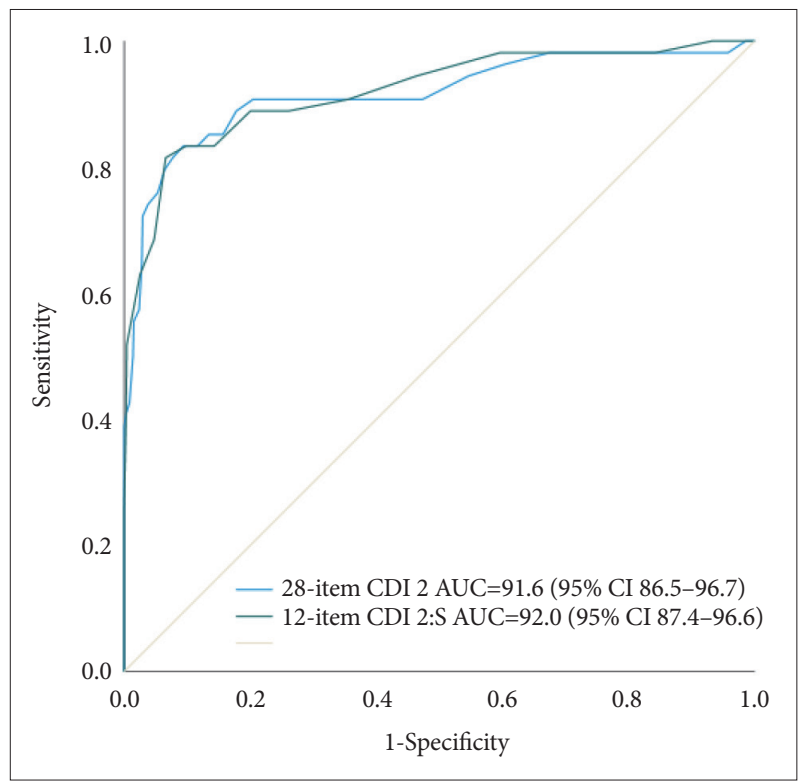

Figure 1. Receiver operating characteristic (ROC) curve for the Children Depression Inventory (CDI) 2 short form and Children Depression Inventory 2. AUC, area under the receiver operating characteristic curve; $\mathrm{Cl}$, confidence interval. sure. Test statistics were all over the acceptable fit (RMSEA= $0.04, \mathrm{CFI}=0.97, \mathrm{TLI}=0.98$ ). Factor loadings were higher than 0.4 for all items in the CDI 2:S. The descriptive statistics and the measurement invariance results are presented in Supplementary Tables 1 and 2 (in the online-only Data Supplement).

\section{DISCUSSION}

The aim of this study was to investigate clinical utility of the Children Depression Inventory 2 short form and determine if the CDI 2:S could perform as well as the CDI 2 to discriminate depressive youth in Korea. The CDI 2:S yielded a good diagnostic accuracy. The cut-off score $\geq 10$ showed an excellent specificity (93\%) and maintained a satisfactory sensitivity (82\%) for the CDI 2:S. The CDI 2 also showed a good diagnostic accuracy with balancing values for sensitivity (83\%) and specificity (91\%) at an optimal cut-off at 20.

This is the first study to investigate the reliability and validity of the CDI 2:S. Although the notion of depression is multifaceted, the author of the CDI and the CDI 2 intended its use as a unidimensional measure. The CFA analysis showed that the CDI 2:S reliably measured 'depression' construct. Factor loadings for most items were greater than 0.4 , suggesting that these items reliably measured the unidimensional construct of depression. This is consistent with previous findings showing that the CDI short version ${ }^{41}$ and the $\mathrm{CDI}^{42}$ should be interpreted as an univocal measure. Ahlen and Ghaderi ${ }^{41}$ tested rigorously tested psychometric properties of the CDI short form (CDI-S) with one-factor, two-factor, and the bi-factor models, and concluded that the CDI-S is a unidimensional measure. They noted that the new short version CDI 2:S would have a stronger predictive validity than does the CDI-S. The

Table 2. Psychometric investigation and clinical validity of the Children Depression Inventory 2

\begin{tabular}{|c|c|c|c|c|c|c|c|c|}
\hline & Sensitivity & Specificity & PPV & NPV & $\mathrm{LR}+$ & LR- & Youden's index & Accuracy \\
\hline 14 & 0.91 & 0.77 & 22.5 & 95.7 & 3.96 & 0.12 & 0.62 & 77 \\
\hline 15 & 0.91 & 0.80 & 19.5 & 95.7 & 4.55 & 0.11 & 0.67 & 80 \\
\hline 16 & 0.89 & 0.82 & 17.6 & 95.7 & 4.94 & 0.13 & $0 ; 70$ & 82 \\
\hline 17 & 0.85 & 0.84 & 15.6 & 95.8 & 5.31 & 0.18 & 0.71 & 84 \\
\hline 18 & 0.85 & 0.87 & 12.7 & 95.8 & 6.38 & 0.20 & 0.70 & 87 \\
\hline 19 & 0.83 & 0.88 & 11.7 & 95.8 & 6.92 & 0.19 & 0.72 & 88 \\
\hline $20^{*}$ & $0.83^{*}$ & $0.91^{*}$ & $8.8^{*}$ & $96.1^{*}$ & $8.11^{*}$ & $0.30^{*}$ & $0.72 *$ & $91^{*}$ \\
\hline 21 & 0.82 & 0.92 & 7.8 & 95.9 & 10.25 & 0.20 & 0.74 & 92 \\
\hline 22 & 0.79 & 0.94 & 5.9 & 95.9 & 13.17 & 0.22 & 0.74 & 94 \\
\hline 23 & 0.76 & 0.95 & 4.9 & 96.0 & 15.20 & 0.25 & 0.73 & 95 \\
\hline 24 & 0.74 & 0.96 & 3.0 & 96.1 & 18.50 & 0.27 & 0.71 & 95 \\
\hline 25 & 0.72 & 0.97 & 2.9 & 96.1 & 24.00 & 0.29 & 0.70 & 96 \\
\hline
\end{tabular}

*the point that maximizes Youden's index. PPV, positive predictive value; NPV, negative predictive value; LR+, positive likelihood ratio; LR-, negative likelihood ratio 
Table 3. Psychometric investigation and clinical validity of the Children Depression Inventory 2 short form

\begin{tabular}{ccccccccc}
\hline & Sensitivity & Specificity & PPV & NPV & LR+ & LR- & Youden's index & Accuracy \\
\hline 6 & 0.89 & 0.74 & 34.8 & 95.7 & 2.55 & 0.14 & 0.55 & 65 \\
7 & 0.89 & 0.80 & 25.5 & 95.7 & 3.41 & 0.15 & 0.63 & 74 \\
8 & 0.83 & 0.86 & 19.6 & 95.7 & 4.44 & 0.14 & 0.69 & 80 \\
9 & 0.83 & 0.90 & 14.0 & 95.8 & 5.83 & 0.19 & 0.69 & 86 \\
$10^{*}$ & $0.82^{*}$ & $0.93^{*}$ & $9.6^{*}$ & $95.8^{*}$ & $8.50^{*}$ & $0.18^{*}$ & $0.74^{*}$ & $90^{*}$ \\
11 & 0.69 & 0.95 & 6.4 & 95.9 & 12.38 & 0.20 & 0.75 & 93 \\
12 & 0.63 & 0.98 & 4.7 & 96.2 & 14.39 & 0.33 & 0.64 & 95 \\
13 & 0.56 & 0.99 & 2.5 & 96.3 & 24.98 & 0.38 & 0.60 & 97 \\
14 & 0.52 & 0.99 & 1.1 & 96.5 & 49.58 & 0.45 & 0.54 & 98 \\
\hline
\end{tabular}

*the point that maximizes Youden's index. PPV, positive predictive value; NPV, negative predictive value; LR+, positive likelihood ratio; LR-, negative likelihood ratio

investigation of the CDI2:S in an independent sample adds further evidence of its validity of scale scores.

Cut-off scores to detect depression in Korean population are known to be somewhat higher than those in Western countries. ${ }^{43}$ Since no previous study has reported the cut-off score for the CDI 2, we reviewed findings of the original version. In a German sample of medically ill children, a 10-item CDI short form was as sensitive as a 26 -item CDI, but was less specific than the full version. ${ }^{20}$ They suggested a cutoff value of 12 for the CDI and a cutoff value of 3 for the CDI:S. On the other hand, in a Korean sample, a score of 20 for the CDI was chosen as the optimal screening cutoff score, with a sensitivity of 0.83 and a specificity of $0.89 .{ }^{43}$ Cultural differences may help explain this phenomenon. Academic achievements seem to put more burden on Asian students than on Western students. ${ }^{44}$ Confucianism places value on education and family dynamics, which may foster academic achievements. ${ }^{45}$ However, it simultaneously creates performance pressure, which leads to subjective feelings of excessive stress and low self-esteem. ${ }^{46} \mathrm{~A}$ recent network analysis study of the CDI in a large sample of school-aged children in Korea ${ }^{47}$ has shown that schoolwork difficulty may cause depression when these children are frustrated with personal achievement in school. Therefore, we postulate that even though not diagnosed with depressive disorders, Korean youths commonly score high in the CDI 2.

As discussed earlier, early detection of childhood depression may improve an individual's life functioning. ${ }^{6}$ Primary health care may play a critical role in opening a door for entry to psychiatric care for people with possible depression. ${ }^{48}$ One empirical study has demonstrated that implementation of an evidence-based depression screening in primary care can improve the screening rate from $0 \%$ to $75 \%$ over a 2 -month project period. ${ }^{49}$ Among those subjects, $6.1 \%$ were diagnosed with depression and started pharmacotherapy. Such a finding calls for an action for primary care providers to adopt evidence- based screening tools. Patients with unknown somatic complaints, sleep disorder, and appetite problem may be at an increased risk of depression. ${ }^{50,51}$ In fact, the prevalence rate before adolescence period is generally low. Many patients with a major depressive disorder start with anxiety symptoms. Those diagnosed with anxiety disorder during childhood, and such anxiety symptoms can develop into depressive symptoms which later meet the criteria for major depressive episode. By screening, primary physicians may refer them to mental health care. We suggest the use of the CDI 2 for a thorough evaluation of emotional symptoms after screening as the CDI 2 has four subscales: negative mood/physical symptoms, negative selfesteem, interpersonal problems, and ineffectiveness. Likewise, clinicians may benefit from a multidimensional assessment when specifying immediate intervention targets.

This study has several limitations. First, since the sampling period was limited to five months, the number of clinical sample with depression was relatively small. This could have reduced statistical power. Second, the clinical sample was recruited from a single tertiary care outpatient clinic, limiting generalizability. Third, inter-rater reliability and test-retest assessment were not analyzed, which may reduce accuracy and coherent validity of the data. Moreover, there was a time gap between the CDI 2 and the K-SADS-PL administration. The administration date was different for each individual due to an outpatient psychiatric setting. Most participants completed the CDI 2, and were interviewed with K-SADS-PL almost one month later. For this short period, possible environmental changes (i.e. increased social support from peers and families) helping them overcome depressive symptoms had occurred. At the same time, stressful events could have worsened depressive symptoms. Furthermore, while we attempted to increase a statistical power by including patients with comorbid psychiatric disorders, sample could be heterogeneous. Depression has high comorbidity in nature, but discriminant validi- 
ty was not explored in the study. Therefore, the results may fail to provide an evidence that the CDI 2:S identify distinct areas of psychopathology in depression. Further validation is required using other self-report, behavioural measures and reports from other respondents such as parents or teachers. Multitrait-multimethod (MTMM) analysis would help increase validity. ${ }^{52}$

In previous studies that investigated the diagnostic utility of the CDI, ${ }^{43}$ participants were recruited from schools or communities. Although they used specific structured interview tools to diagnose depression, participants were originally sampled from non-clinical settings. This sampling bias might contribute to false positives, with possibility of subjects being misclassified as MDD. However, in our research, a large sample of children and adolescents was used and every subject in the clinical group underwent rigorous structured interview by clinical psychologists. The power of psychometric properties of the CDI 2:S in diagnostic validity was based on a structured diagnostic interview, which increases methodological power.

To conclude, this is an initial study reporting sensitivity, specificity, and AUC parameters of the CDI 2 cut-off scores. Our study confirmed the use of both the CDI 2 and the CDI 2:S in a psychiatric setting in Korea. Although the short form was suggested to use as a screening tool in primary care settings, only a few studies have examined the utility of the CDI short form. ${ }^{41}$ We expect the use of the CDI 2 and the CDI 2:S in various settings. It might be used as a screening tool for a group of youth to determine what proportion of students may require further psychological evaluations. Also, teachers may use the CDI 2:S to detect depressive students to further refer them to clinics and allow them to benefit from further support. In clinical settings, CDI 2:S can be used as a 5-minute quick screening tool. Our results suggest that the 12-item CDI $2: S$ is an attractive alternative to the 28 -item CDI 2 in a timelimited psychiatric outpatient setting. As a screening tool, those with positive screening results should further take clinical interviews to rule out false positives and verify depression diagnoses.

\section{Supplementary Materials}

The online-only Data Supplement is available with this article at https://doi.org/10.30773/pi.2021.0296.

\section{Availability of Data and Material}

The datasets generated or analyzed during the study are available from the corresponding author on reasonable request.

\section{Conflicts of Interest}

The authors have no potential conflicts of interest to disclose.

\section{Author Contributions}

Conceptualization: Yaehee Cho, Eun-Ho Lee, Ji-Hae Kim. Data curation: Sang-Hwang Hong, Ji-Hae Kim, Yoo-Sook Joung. Formal analysis: Yaehee
Cho. Writing_original draft: Yaehee Cho. Writing_review \& editing: Eun-Ho Lee, Ji-Hae Kim, Yoo-Sook Joung.

\section{ORCID iDs}

Yaehee Cho

Eun-Ho Lee

Sang-Hwang Hong

Yoo-Sook Joung

Ji-Hae Kim https://orcid.org/0000-0001-7577-4842

https://orcid.org/0000-0003-2550-8225

https://orcid.org/0000-0002-2433-5564

https://orcid.org/0000-0002-9225-4643

https://orcid.org/0000-0003-1037-1801

\section{Funding Statement None}

\section{Acknowledgments}

The authors would like to pay our gratitude and our respects to Soon-Taeg Hwang. After helping data collection, he passed away in August 24th 2018.

\section{REFERENCES}

1. Liu Q, He H, Yang J, Feng X, Zhao F, Lyu J. Changes in the global burden of depression from 1990 to 2017: Findings from the Global Burden of Disease study. J Psychiatr Res 2020;126:134-140.

2. Benjet C, Albor YC, Bocanegra ES, Borges G, Méndez E, Casanova L, et al. Incidence and recurrence of depression from adolescence to early adulthood: a longitudinal follow-up of the Mexican Adolescent Mental Health Survey. J Affect Disord 2020;263:540-546.

3. Fergusson DM, Woodward LJ. Mental health, educational, and social role outcomes of adolescents with depression. Arch Gen Psychiatry 2002; 59:225-231.

4. Weersing VR, Shamseddeen W, Garber J, Hollon SD, Clarke GN, Beardslee WR, et al. Prevention of Depression in At-Risk Adolescents: Predictors and Moderators of Acute Effects. J Am Acad Child Adolesc Psychiatry 2016;55:219-226.

5. Stockings E, Degenhardt L, Lee YY, Mihalopoulos C, Liu A, Hobbs M, et al. Symptom screening scales for detecting major depressive disorder in children and adolescents: a systematic review and meta-analysis of reliability, validity and diagnostic utility. J Affect Disord 2015;174:447463.

6. Clayborne ZM, Varin M, Colman I. Systematic review and meta-analysis: adolescent depression and long-term psychosocial outcomes. J Am Acad Child Adolesc Psychiatry 2019;58:72-79.

7. Johnson D, Dupuis G, Piche J, Clayborne Z, Colman I. Adult mental health outcomes of adolescent depression: a systematic review. Depress Anxiety 2018;35:700-716.

8. Forman-Hoffman VL, Viswanathan M. Screening for depression in pediatric primary care. Curr Psychiatry Rep 2018;20:62.

9. Rice F, Riglin L, Lomax T, Souter E, Potter R, Smith DJ, et al. Adolescent and adult differences in major depression symptom profiles. J Affect Disord 2019;243:175-181.

10. Loades ME, Chatburn E, Higson-Sweeney N, Reynolds S, Shafran R, Brigden A, et al. Rapid Systematic Review: The Impact of Social Isolation and Loneliness on the Mental Health of Children and Adolescents in the Context of COVID-19. J Am Acad Child Adolesc Psychiatry 2020; 59:1218-1239.e1213.

11. Harder VS, Barry SE, French S, Consigli AB, Frankowski BL. Improving adolescent depression screening in pediatric primary care. Acad Pediatr 2019;19:925-933.

12. Maurer DM, Raymond TJ, Davis BN. Depression: screening and diagnosis. Am Fam Physician 2018;98:508-515.

13. Cohen JR, So FK, Young JF, Hankin BL, Lee BA. Youth depression screening with parent and self-reports: assessing current and prospective depression risk. Child Psychiatry Hum Dev 2019;50:647-660.

14. Bernaras E, Jaureguizar J, Garaigordobil M. Child and adolescent depression: a review of theories, evaluation instruments, prevention pro- 
grams, and treatments. Front Psychol 2019;10:543.

15. Finch AJ Jr, Saylor CF, Edwards GL. Children's depression inventory: sex and grade norms for normal children. J Consult Clin Psychol 1985; 53:424-425.

16. Smith M, Calam R, Bolton C. Psychological factors linked to self-reported depression symptoms in late adolescence. Behav Cogn Psychother 2009;37:73-85.

17. Abdel-Khalek AM. Criterion-related validity of the Arabic Children's Depression Inventory. Psychol Rep 1998;82:930.

18. Davanzo P, Kerwin L, Nikore V, Esparza C, Forness S, Murrelle L. Spanish translation and reliability testing of the Child Depression Inventory. Child Psychiatry Hum Dev 2004;35:75-92.

19. Roelofs J, Braet C, Rood L, Timbremont B, van Vlierberghe L, Goossens $\mathrm{L}$, et al. Norms and screening utility of the Dutch version of the Children's Depression Inventory in clinical and nonclinical youths. Psychol Assess 2010;22:866-877.

20. Allgaier AK, Frühe B, Pietsch K, Saravo B, Baethmann M, Schulte-Körne G. Is the Children's Depression Inventory Short version a valid screening tool in pediatric care? A comparison to its full-length version. J Psychosom Res 2012;73:369-374.

21. Giannakopoulos G, Kazantzi M, Dimitrakaki C, Tsiantis J, Kolaitis G, Tountas Y. Screening for children's depression symptoms in Greece: the use of the Children's Depression Inventory in a nation-wide school-based sample. Eur Child Adolesc Psychiatry 2009;18:485-492.

22. Poli P, Sbrana B, Marcheschi M, Masi G. Self-reported depressive symptoms in a school sample of Italian children and adolescents. Child Psychiatry Hum Dev 2003;33:209-226.

23. Rosliwati M, Rohayah H, Jamil B, Zaharah S. Validation of the Malay version of Children Depression Inventory (CDI) among children and adolescents attending outpatient clinics in Kota Bharu, Kelantan. Malays J Psychiatry 2008;17:23-29.

24. Yu D, Li X. Preliminary use of the Children's Depression Inventory (CDI) in China. J Chin Mental Health 2000;14:225-227.

25. Ozono S, Nagamitsu S, Matsuishi T, Yamashita Y, Ogata A, Suzuki S, et al. Reliability and validity of the Children's Depression Inventory-Japanese version. Pediatr Int 2019;61:1159-1167.

26. Kovacs M. Children's depression inventory (CDI and CDI 2). Encyclopedia Clin Psychol 2014:1-5.

27. Kim MH, Mazenga AC, Devandra A, Ahmed S, Kazembe PN, Yu X, et al. Prevalence of depression and validation of the Beck Depression Inventory-II and the Children's Depression Inventory-Short amongst HIV-positive adolescents in Malawi. J Int AIDS Soc 2014;17:18965.

28. Kim HJ, Lee EH, Hwang ST, Hong SH, Kim JH. Psychometric properties of the children's depression inventory- 2 among a community-based sample of Korean children and adolescents. Korean J Clin Psychol 2018; 37:177-186.

29. Kaufman J, Birmaher B, Brent D, Rao U, Flynn C, Moreci P, et al. Schedule for Affective Disorders and Schizophrenia for School-Age Children-Present and Lifetime Version (K-SADS-PL): initial reliability and validity data. J Am Acad Child Adolesc Psychiatry 1997;36:980-988.

30. Kim YS, Cheon KA, Kim BN, Chang SA, Yoo HJ, Kim JW, et al. The reliability and validity of kiddie-schedule for affective disorders and schizophrenia-present and lifetime version-Korean version (K-SADSPL-K). Yonsei Med J 2004;45:81-89.

31. Hanley JA, McNeil BJ. The meaning and use of the area under a receiver operating characteristic (ROC) curve. Radiol 1982;143:29-36.

32. Youden WJ. Index for rating diagnostic tests. Cancer 1950;3:32-35.

33. Rosseel Y. lavaan: An R package for structural equation modeling and more. Version 0.5-12 (BETA). J Statist Softw 2012;48:1-36.

34. Chen PY, Wu W, Garnier-Villarreal M, Kite BA, Jia F. Testing measurement invariance with ordinal missing data: a comparison of estimators and missing data techniques. Multivar Behav Res 2020;55:87-101.

35. Kline RB. Principles and Practice of Structural Equation Modeling. New York: Guilford Publications; 2015.

36. Tucker LR, Lewis C. A reliability coefficient for maximum likelihood factor analysis. Psychometrika 1973;38:1-10.

37. Byrne BM, Shavelson RJ, Muthén B. Testing for the equivalence of factor covariance and mean structures: the issue of partial measurement invariance. Psychol Bull 1989;105:456.

38. Hu Lt, Bentler PM. Cutoff criteria for fit indexes in covariance structure analysis: conventional criteria versus new alternatives. Struct Equ Modeling 1999;6:1-55.

39. Brown MW, Cudeck R. Alternative ways of assessing model fit. Test Struct Equ Models 1993;154:136-162.

40. Twenge JM, Nolen-Hoeksema S. Age, gender, race, socioeconomic status, and birth cohort differences on the children's depression inventory: a meta-analysis. J Abnorm Psychol 2002;111:578-588.

41. Ahlen J, Ghaderi A. Evaluation of the Children's Depression InventoryShort Version (CDI-S). Psychol Assess 2017;29:1157-1166.

42. Stumper A, Olino TM, Abramson LY, Alloy LB. A factor analysis and test of longitudinal measurement invariance of the Children's Depression Inventory (CDI) across adolescence. J Psychopathol Behav Assess 2019;41:692-698.

43. Bang YR, Park JH, Kim SH. Cut-off scores of the children's depression inventory for screening and rating severity in Korean adolescents. Psychiatry Investig 2015;12:23-28.

44. Lee EH, Lee SJ, Hwang ST, Hong SH, Kim JH. Reliability and validity of the beck depression inventory-II among Korean adolescents. Psychiatry Investig 2017;14:30-36.

45. Pinquart M, Kauser R. Do the associations of parenting styles with behavior problems and academic achievement vary by culture? Results from a meta-analysis. Cultur Divers Ethnic Minor Psychol 2018;24:75100.

46. Soenens B, Park SY, Vansteenkiste M, Mouratidis A. Perceived parental psychological control and adolescent depressive experiences: a crosscultural study with Belgian and South-Korean adolescents. J Adolesc 2012;35:261-272.

47. Kim D, Kwon HJ, Ha M, Lim MH, Kim KM. Network analysis for the symptom of depression with Children's Depression Inventory in a large sample of school-aged children. J Affect Disord 2021;281:256-263.

48. Davey CG, McGorry PD. Early intervention for depression in young people: a blind spot in mental health care. Lancet Psychiatry 2019;6:267272.

49. Bose J, Zeno R, Warren B, Sinnott LT, Fitzgerald EA. Implementation of universal adolescent depression screening: quality improvement outcomes. J Pediatr Health Care 2021;35:270-277.

50. Allgaier AK, Krick K, Opitz A, Saravo B, Romanos M, Schulte-Körne G. Improving early detection of childhood depression in mental health care: the Children's Depression Screener (ChilD-S). Psychiatry Res 2014; 217:248-252.

51. Agnafors S, Norman Kjellström A, Torgerson J, Rusner M. Somatic comorbidity in children and adolescents with psychiatric disorders. Eur Child Adolesc Psychiatry 2019;28:1517-1525.

52. Eid M, Geiser C, Nussbeck FW. Multitrait-multimethod analysis in psychotherapy research: new methodological approaches. Psychother Res 2009;19:390-396. 
Supplementary Table 1. Descriptive statistics of the CDI 2:S

\begin{tabular}{rl}
\hline & Mean (SD) \\
\hline Item 1 & $0.17(0.426)$ \\
Item 2 & $0.58(0.575)$ \\
Item 3 & $0.39(0.531)$ \\
Item 4 & $0.41(0.531)$ \\
Item 5 & $0.17(0.434)$ \\
Item 6 & $0.24(0.507)$ \\
Item 7 & $0.62(0.592)$ \\
Item 8 & $0.68(0.566)$ \\
Item 9 & $0.47(0.671)$ \\
Item 10 & $0.63(0.715)$ \\
Item 11 & $0.27(0.505)$ \\
Item 12 & $0.28(0.525)$ \\
\hline
\end{tabular}

SD, standard deviation; CDI 2:S, Children Depression Inventory 2 short form 
Supplementary Table 2. Tests of measurement invariance of the CDI 2:S across gender

\begin{tabular}{lccccccccc}
\hline \multicolumn{1}{c}{ Model } & S-B $\chi^{2}$ & df & CFI & $\Delta$ CFI & RMSEA & $\Delta$ RMSEA & TLI & $\Delta$ TLI \\
\hline Model 1: configural & 141.752 & 108 & 0.995 & - & 0.028 & - & 0.994 & - \\
Model 2: metric & 160.176 & 119 & 0.994 & 0.001 & 0.030 & 0.002 & 0.993 & 0.001 \\
Model 3: scalar & 204.164 & 130 & 0.990 & 0.004 & 0.039 & 0.009 & 0.990 & 0.003 \\
\hline
\end{tabular}

S-B $\chi^{2}$, Satorra-Bentler scaled $\chi^{2}$; df, degree of freedom; CFI, comparative fit index; RMSEA, root mean square error of approximation; TLI, Tucker-Lewis Index; CDI 2:S, Children Depression Inventory 2 short form 